\title{
Low periostin expression predicts poor survival in intestinal type gastric cancer patients
}

This article was published in the following Dove Press journal:

Cancer Management and Research

\section{Teming Zhang, ',* Zheng Han, 1,* Arvine Chandoo, 1,* Xincheng Huang,' Xiangwei Sun,' Lele Ye, ${ }^{2}$ Changyuan $\mathrm{Hu},{ }^{3}$ Xiangyang Xue, ${ }^{2}$ Yingpeng Huang,' Xian Shen,' Wenjun Chang, ${ }^{4}$ Xiaoming Lin $^{5}$ \\ 'Department of General Surgery, Second Affiliated Hospital, Wenzhou Medical University, Wenzhou, People's Republic of China; ${ }^{2}$ Department of Microbiology and Immunology, Institute of Molecular Virology and Immunology, Institute of Tropical Medicine, Wenzhou Medical University, Wenzhou, People's Republic of China; ${ }^{3}$ Department of Gastrointestinal Surgery, The First Affiliated Hospital, Wenzhou Medical University, Wenzhou, People's Republic of China; ${ }^{4}$ Department of Environmental Hygiene, Second Military Medical University, Shanghai People's Republic of China; ${ }^{5}$ Department of Thoracic Surgery, The First Affiliated Hospital of Wenzhou Medical University, Nanbaixiang, Wenzhou, People's Republic of China}

*These authors contributed equally to this work

Correspondence: Xian Shen Second Affiliated Hospital, Wenzhou Medical University, No. 109 Xueyuan Road, Wenzhou, Zhejiang 325003,

People's Republic of China

Email 13968888872@I63.com

Xiaoming Lin

Department of Thoracic Surgery, The First Affiliated Hospital of Wenzhou Medical University, Nanbaixiang, Ouhai District,

Wenzhou, Zhejiang 325003, China Email sheermanlin@I26.com
Background and aim: Periostin is a protein from the Fascilin family. It is commonly present in normal tissues and is responsible for cell adhesion. Evidence has emerged showing that changes in periostin expression play an important role in tumor initiation, development, and progression. This study aims to investigate the effect of periostin in gastric cancer (GC) patients who underwent gastrectomy. Seven hundred and forty-seven GC patients who underwent gastrectomy between December 2006 and July 2011 were included in this study.

Methods: Seven hundred and forty-seven cancer tissues and 70 paired adjacent normal tissues were collected. Periostin expression was evaluated by immunohistochemistry. The Gene Expression Omnibus database was used to study the association between the mRNA level and patient's overall survival. The tumor microenvironment was also studied.

Results: Periostin expression in stroma was downregulated in tumor tissues but it was upregulated in the epithelial cells. After dividing the tissues according to the Lauren Classification, we found that periostin expression in stroma and epithelial cells was higher in intestinal type than in diffuse type $(P<0.001$ and $P=0.010$, respectively). Periostin was an independent predictor of lymph node (LN) metastasis in GC patients. The study of CD163(+) tumor-associated macrophages (TAMs) revealed that in diffuse type GC, periostin expression was associated with CD163(+) TAMs.

Conclusion: We found that the periostin expression can predict LN metastasis in patients undergoing curative gastrectomy. Intestinal type GC patients with high periostin level had both a favorable survival and lesser LN metastasis.

Keywords: lymph node metastasis, tumor microenvironment, tissue microarray, cell adhesion, Lauren Classification

\section{Background}

Gastric cancer (GC) is the second most common cause of cancer-related deaths worldwide. ${ }^{1}$ There are improvements in the treatment of GC, but the survival of these patients is still dismal, with a 5 -year overall survival of less than $20 \%$ of patients. ${ }^{1}$ Development in proteomics might help us to better understand the pathological pathways of GC and identify the targets that are more promising. ${ }^{2}$ There exist several classifications for GC, and one of them is Lauren Classification. Under Lauren Classification, GC can be divided into intestinal and diffused types. ${ }^{3}$ It has been found that tumor cells in the diffused type GC lack cell adhesion and infiltrate the stroma as a single cell or as a small subgroup, which lead to a population of scattered tumor cells. Arranged in tubular or glandular formations, intestinal tumor cells exhibit adhesion and have a better survival than diffused type GC. ${ }^{4}$

Extracellular matrix (ECM) proteins mainly maintain the tissue structure. Some ECM proteins, for example, matricellular proteins, secrete protein acidic, rich cysteine, 
osteoponin, and thrombospondin. ${ }^{5}$ They affect cell function by modulating cell interactions. Periostin, a matricellular protein, belongs to the Fascilin family. ${ }^{6-8}$ Periostin also known as osteoblast-specific factor 2 is commonly expressed in many normal tissues. ${ }^{9}$ It is highly homologous to the beta ig-h3, ie, one of the members of the Fascilin-I protein family, which is a TGF-beta-responsive gene. ${ }^{10,11}$ Beta ig-h3 is responsible for cell adhesion and fibroblasts spreading through integrin alpha-b26, and it suppresses CHO cells to develop into nude mice. $^{12}$

Periostin is overexpressed by TGF-b3 and is suppressed by Wnt-3 by inhibiting the glycogen kinase $3 b$ (GSK-3b). ${ }^{13}$ Overexpression of periostin gene has been found in lung cancer, which correlated with the poor survival. ${ }^{14,15}$ Periostin was present at a higher level in stage IV thymoma than in stage I thymoma, which suggests that an increase in the expression of periostin may be associated with the tumor progression. ${ }^{16}$ Recently, several studies proved that periostin plays an important role in the occurrence and development of many tumors, such as colon, esophagus, pancreas, lung, ovary, and prostate cancers. ${ }^{17-21}$ Periostin causes these tumors to advance, proliferate, and metastasize and results in epithelial-mesenchymal transformation, but the role of periostin in $\mathrm{GC}$ is still uncertain. There are several factors that affect the survival of GC patients; lymph nodes (LNs) metastasis is one among them. ${ }^{22}$ The National Comprehensive Cancer Network (NCCN) guidelines recommend the perioperative use of chemotherapy or chemoradiotherapy in LN-positive patients. ${ }^{23}$ For preoperative use of chemotherapy or chemoradiotherapy, accurate evaluation of LNs metastasis is important.22

A study showed that in GC, the extracellular periostin was markedly downregulated in the tumor tissue than in the normal tissue. ${ }^{19}$ It was also reported that periostin as a niche component enhanced the tumor development by activating the ERK pathway. ${ }^{24}$ The epithelial cell-derived periostin stabilized p53 and E-cadherin proteins through the $\mathrm{Rb} / \mathrm{E} 2 \mathrm{~F} 1 /$ p14ARF/Mdm2 signaling pathway and suppressed GC. ${ }^{25}$ Tumor microenvironment plays an important role in tumor development and metastasis. ${ }^{26}$ The main component of the tumor microenvironment is tumor-associated macrophage (TAM). TAM is a M2-like phenotype, and the CD163 is a marker specific for M2 macrophage. Periostin have an effect in recruiting CD163(+) TAMs in glioma, ovarian cancer, and other diseases. ${ }^{27-32}$

There are few researches that studied the expression and effect of cellular or stromal periostin in GC. On the basis of the above findings, we investigated the expression of cellular and stromal periostin in tumor and normal tissues. The aim of this study was to evaluate the correlation between the expression of periostin in tumor tissues and LN metastasis and clinical characteristics in GC patients, and the influence of periostin on CD163(+) TAMs.

\section{Patients and methods}

Patients and specimens

Seven hundred forty-seven patients in the Second Affiliated Hospital of Wenzhou Medical University (Wenzhou, People's Republic of China), who underwent surgical resection for GC between December 2006 and July 2011, were included in this study. All the gastric adenocarcinoma tissues and 70 paired adjacent normal tissues (at least $10 \mathrm{~cm}$ from the negative margin) were formalin fixed paraffin embedded (FFPE) and confirmed by histopathologic analysis. This study consisted of 518 male and 204 female patients, whose age ranged from 20 to 86 years (median, 59 years). The demographic and clinicopathological characteristics such as age, gender, differentiation status, Lauren type, serum carcinoembryonic antigen (CEA), carbohydrate antigen 19-9 (CA 19-9) and carbohydrate antigen 72-4 (CA 72-4) levels, depth of invasion, LN metastasis, and TNM stage at the time of surgery (TNM stage was classified according to the American Joint Committee on Cancer Staging Manual eighth edition) were included. The Review Board of the Second Affiliated Hospital of Wenzhou Medical University (Zhejiang, People's Republic of China) approved this study. The tissue microarray (TMA) was constructed as described previously. ${ }^{33}$ All patients were aware of the research and signed the informed consent form.

\section{Immunohistochemistry (IHC)}

IHC detection in TMA was performed manually. Briefly, the sections were dewaxed by incubating in dimethyl benzene at $45^{\circ}$ for 60 minutes, followed by immersion in distilled water. Endogenous peroxidase activity was inhibited by incubating in a $0.5 \%$ hydrogen peroxide bath for 10 minutes. After washing three times with 0.01 M PBS (pH 7.4), the slides were immersed in citrate antigen retrieval buffer (Zhongshan Golden Bridge Biotechnology, Beijing, People's Republic of China). After blocking in sheep serum for 30 minutes, the sections were incubated with anti-POSTN antibody (HPA012306; Sigma Chemical Co., St. Louis, MO, USA; diluted 1:200) in a humidified chamber at $24^{\circ} \mathrm{C}$ for 2 hours. After washing three times with PBS, Dako EnVision FLEX detection system (Dako, Carpinteria, CA, USA) was used for visualization of the staining results according to the manufacturer's instructions. Slides were then counterstained using hematoxylin, dehydrated, and sealed with neutral gum. 


\section{Detection of periostin expression}

The TMA was processed in a flow as shown in Figure 1. The total view of the TMA, which was obtained by a digital slice scanner (Easyscan6; MOTIC Medical Diagnostic Systems, Fuzhou, People's Republic of China), was separated into single spots, followed by eliminating microarray spots with no tumor tissue, missing spots, and spots with little valid area. The rest spots were estimated if the epithelial cells were periostin positive (percentage of positive cells $\geq 5 \%$ ) or negative. In case of epithelial cell-negative tissue, a computer-automated method was conducted to evaluate the expression of periostin in stroma (Image pro plus 6.0; Media Cybernetics Inc.). Finally, average optical density (AOD; integrated optical density/total area) of the stromal periostin expression in epithelial cellnegative tissue was calculated. The TMAs stained for CD163 were processed in a similar method, and the expression level was represented as positive area ratio (positive area/total area).

\section{Bioinformatics analysis of Gene Expression Omnibus (GEO) data sets}

Periostin expression data for mRNA level and clinical information for GC available in GEO (http://www.ncbi.nlm. nih.gov/geo/) database were used to investigate the association between periostin expression level and the overall survival of GC patients. Data from six available GEO data sets, GSE14210 ( $\mathrm{n}=146)$, GSE15459 ( $\mathrm{n}=200)$, GSE22377 $(\mathrm{n}=43), \operatorname{GSE} 29272$ (268), GSE51105 ( $\mathrm{n}=94)$, and GSE62254 $(\mathrm{n}=300)$, were obtained. Eight hundred seventy-six patients with detailed information were included in the analysis.
Univariate survival analysis and Kaplan-Meier analysis with a log-rank test were used to construct survival curves. All percentiles between the first and third quartiles were computed, and the best performing threshold was used as a cutoff value to sort all the patients into low- and high-expression groups. All the bioinformatics analyses were performed using Kaplan-Meier Plotter (http://kmplot.com/analysis/).

\section{Statistical analyses}

Chi-squared test was used to compare the positive rate of periostin in epithelial cells among different kinds of tissues. Mann-Whitney $U$ test was performed to compare AOD of stromal periostin expression level in epithelial cell-negative tissue among different kinds of tissues. The cutoff value of AOD of stromal periostin expression in epithelial cellnegative tissue was determined by overall survival-specific receiver operating curve (ROC) analysis according to the Youden index. The patients of epithelial cell-negative group were then divided into high-expression and low-expression subgroups.

A comparison among the clinicopathological variables was made using the chi-squared test. After univariate analysis, variables with $P$-value $<0.05$ were included in the multivariate logistic regression analysis to confirm independent variables. The forward stepwise method was used to eliminate variables that did not yield significant information. The HR and $95 \%$ CI of each independent variable were calculated. Univariate survival analysis and Kaplan-Meier's analysis with a log-rank test were performed to construct survival curves. Mann-Whitney $U$ test was performed to compare

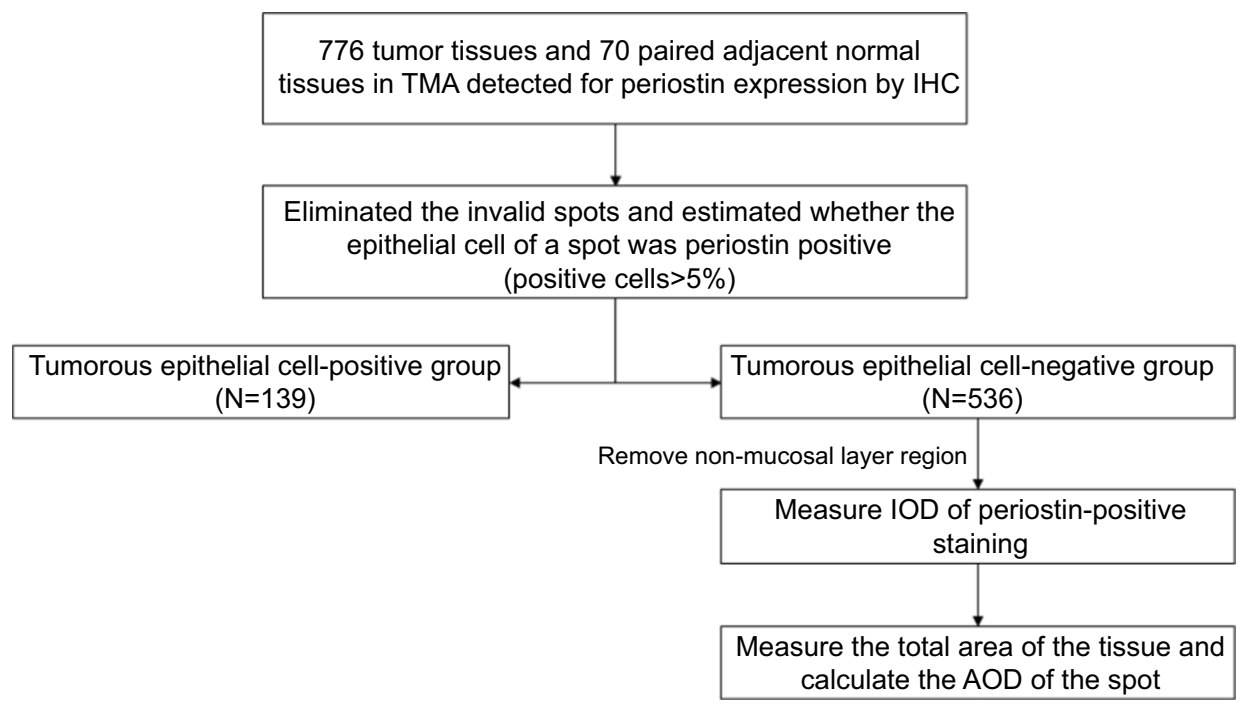

Figure I The process flow of each spot in the TMA.

Abbreviations: AOD, average optical density; IHC, immunohistochemistry; IOD, integrated optical density; TMA, tissue microarray. 
the expression of CD163 in different GC tissues. Statistical analyses were performed using R and SPSS V.23 for Windows (SPSS Inc., Chicago, IL, USA). Statistical significance was defined as $P<0.05$.

\section{Results}

\section{Cellular and stromal periostin was differentially expressed in GC and adjacent normal tissues}

IHC was used to determine the periostin expression in GC tissue and adjacent normal tissue. Periostin was expressed in the stroma of all the tissues at various levels (Figure 2B, C, E, $\mathrm{F}, \mathrm{H}$, and J). The part of tissues even showed an intracellular staining pattern in epithelial cells (Figure 2A, D, G, and I). Six hundred seventy-five tumor tissues and 69 adjacent normal tissues were used for analysis. The positive proportion of periostin in epithelial cells of tumor tissue was $20.6 \%$ (139 of 675), which is much higher than $8.7 \%$ (6 of $69, P=0.016$ ) in epithelial cells of the adjacent normal tissue (Figure $2 \mathrm{~K}$ ). In all the periostin epithelial cell-negative tissues, AOD of stromal periostin expression in adjacent normal tissue was significantly higher than that in GC tissue $(P<0.001$; Figure 2L). The expression of periostin in different types of gastric tissues varied. A total of $27.2 \%(91 / 338)$ was positive in intestinal type GC tissue, which was significantly higher than the diffuse type GC tissue $(13.7 \%, 45 / 328, P<0.001$; Figure $2 \mathrm{M})$. AOD of stromal periostin expression in intestinal type GC tissue was significantly higher than that in the diffuse type GC tissue ( $P=0.010$; Figure $2 \mathrm{~N}$ ).

\section{Periostin expression is associated with lesser LN metastasis}

There was a statistical significance between periostin epithelial cell-positive and periostin epithelial cell-negative groups with respect to gender $(P=0.016)$, differentiation status $(P<0.001)$, Lauren type $(P<0.001)$, depth of invasion $(P=0.008), \mathrm{LN}$ involvement $(P<0.001)$, and TNM stage $(P=0.003)$. For the expression of stromal periostin, a similar difference in depth of invasion $(P<0.001)$, LN involvement $(P=0.002)$, and TNM stage $(P<0.001)$ were observed (Table 1). Univariate analysis showed that cellular and stromal periostin, age, diameter, Lauren type, serum

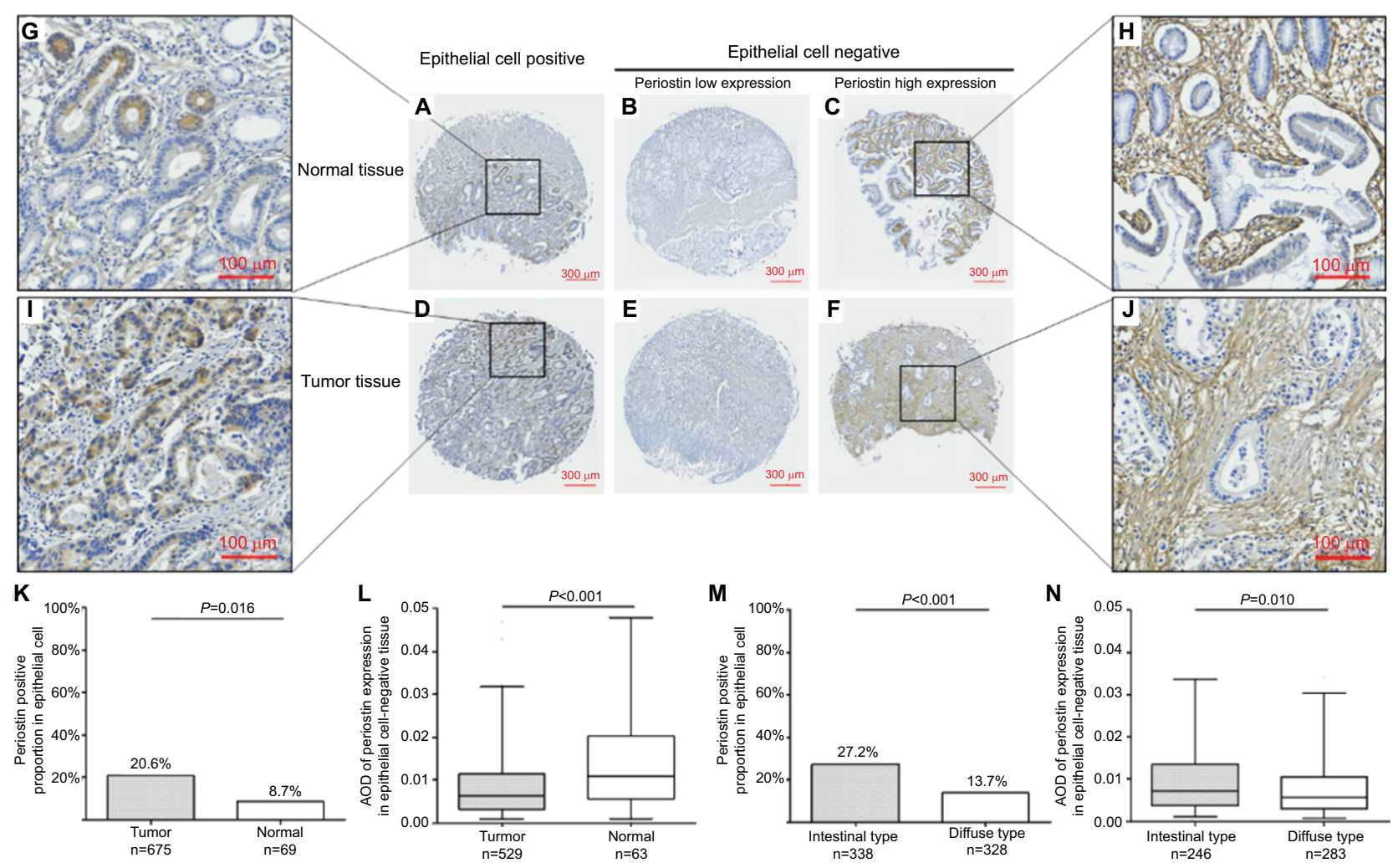

Figure 2 Periostin expression in GC and adjacent normal tissue.

Notes: (A, D, G, and I) Periostin staining in epithelial cell-positive normal and tumor tissues. (B and E) Low expression of periostin in epithelial cell-negative normal and tumor tissues. (C, F, H, and J) High expression of periostin in epithelial cell-negative normal and tumor tissue. (K and $\mathbf{L})$ Differential expression of cellular and stromal periostin between tumor and adjacent normal tissue. ( $\mathbf{M}$ and $\mathbf{N}$ ) Differential expression of cellular and stromal periostin between intestinal type and diffuse type GC. Abbreviations: AOD, average optical density; GC, gastric cancer. 
Table I The clinical characteristics of patients according to periostin expression

\begin{tabular}{|c|c|c|c|c|c|c|}
\hline \multirow[t]{2}{*}{ Variables } & \multicolumn{3}{|c|}{$\begin{array}{l}\text { Periostin expression in epithelial cells } \\
\text { of tumor tissue }\end{array}$} & \multicolumn{3}{|c|}{$\begin{array}{l}\text { Periostin expression level in epithelial } \\
\text { cell-negative tumor tissue }\end{array}$} \\
\hline & Negative $(n=536)$ & Positive $(n=139)$ & $P$-value & Low $(n=371)$ & High $(n=164)$ & $P$-value \\
\hline Age (years), n (\%) & & & 0.098 & & & 0.155 \\
\hline$<60$ & $275(53.9)$ & $61(45.9)$ & & $18 \mid(5 \mid .7)$ & $93(58.5)$ & \\
\hline$\geq 60$ & $235(46.2)$ & $72(53.7)$ & & $169(48.3)$ & $66(41.5)$ & \\
\hline Gender, n (\%) & & & 0.018 & & & 0.288 \\
\hline Male & $358(69.1)$ & $108(79.4)$ & & $25 I(70.5)$ & $106(65.8)$ & \\
\hline Female & $160(30.9)$ & $28(20.6)$ & & $105(29.5)$ & $55(34.2)$ & \\
\hline Differentiation status, $\mathrm{n}(\%)$ & & & $<0.001$ & & & 0.831 \\
\hline Well and moderate & $147(3 \mid . I)$ & $62(47.7)$ & & I0I (30.7) & $45(31.7)$ & \\
\hline Poor and undifferentiated & $325(68.9)$ & $68(52.3)$ & & $228(69.3)$ & $97(68.3)$ & \\
\hline Lauren type, $\mathrm{n}(\%)$ & & & $<0.001$ & & & 0.068 \\
\hline Intestinal type & $247(46.6)$ & $91(66.9)$ & & $161(43.9)$ & $85(52.5)$ & \\
\hline Diffuse type & $283(53.4)$ & $45(33.1)$ & & $206(56.1)$ & $77(47.5)$ & \\
\hline Serum CEA (ng/mL), n (\%) & & & 0.538 & & & 0.305 \\
\hline$<5$ & $406(82.5)$ & $10 \mathrm{I}(80.2)$ & & $273(8 I .5)$ & $133(85.3)$ & \\
\hline$\geq 5$ & $86(I 7.5)$ & $25(19.8)$ & & $62(18.5)$ & $23(14.7)$ & \\
\hline Serum CAI9-9 (U/mL), n (\%) & & & 0.755 & & & 0.481 \\
\hline$<37$ & $394(83.8)$ & $102(85.0)$ & & $263(83.0)$ & $130(85.5)$ & \\
\hline$\geq 37$ & $76(16.2)$ & $18(15.0)$ & & $54(17.0)$ & $22(14.5)$ & \\
\hline Serum CA72-4 (U/mL), n (\%) & & & 0.934 & & & 0.782 \\
\hline$<6.9$ & $295(78.2)$ & $70(78.7)$ & & $196(77.8)$ & $98(79.0)$ & \\
\hline$\geq 6.9$ & $82(21.8)$ & $19(21.3)$ & & $56(22.2)$ & $26(21.0)$ & \\
\hline Depth of invasion, $\mathrm{n}(\%)$ & & & 0.006 & & & $<0.001$ \\
\hline $\mathrm{TI}+\mathrm{T} 2$ & $176(34.0)$ & $63(46.7)$ & & $103(28.9)$ & $73(45.3)$ & \\
\hline $\mathrm{T} 3+\mathrm{T} 4$ & $342(66.0)$ & $72(53.3)$ & & $253(7 I . I)$ & $88(54.7)$ & \\
\hline Lymph node involvement, n (\%) & & & $<0.001$ & & & 0.002 \\
\hline No & $185(35.7)$ & 7I (52.2) & & $111(3 I .2)$ & $73(45.3)$ & \\
\hline Yes & $333(64.3)$ & $65(47.8)$ & & $245(68.8)$ & $88(54.7)$ & \\
\hline TNM stage, n (\%) & & & 0.002 & & & $<0.001$ \\
\hline $\mathrm{I}+\mathrm{II}$ & $238(45.9)$ & $82(60.7)$ & & $145(40.7)$ & $93(57.8)$ & \\
\hline III+IV & $280(54.1)$ & $53(39.3)$ & & $211(59.3)$ & $68(42.2)$ & \\
\hline
\end{tabular}

Abbreviations: CA19-9, carbohydrate antigen 19-9; CA72-4, carbohydrate antigen 72-4; CEA, carcinoembryonic antigen.

CEA, serum CA19-9, serum CA72-4, depth of invasion, and differentiation status were potential factors for the $\mathrm{LN}$ involvement $(P<0.005$; Table S1). In multivariate analysis, we found that these factors were independent predictors of LN metastasis $(P<0.05)$. We also found that periostin is a protective factor (Table 2 ).

\section{Loss of periostin expression is associated with worse survival of GC patients}

Kaplan-Meier survival analysis was performed in patients with different periostin expression levels in epithelial cells and stromal of tumor tissue. Our analysis revealed that the patients with periostin epithelial cell-positive GC had a favorable survival compared to those with periostin epithelial cell-negative GC $(P=0.003, \mathrm{HR}=0.600,95 \%$ $\mathrm{CI}=0.428-0.841$; Figure $3 \mathrm{~A})$. Similarly, the stromal peri- ostin high-expression group had a longer overall survival relative to the low-expression group $(P=0.004, \mathrm{HR}=0.638$, $95 \% \mathrm{CI}=0.468-0.870$; Figure $3 \mathrm{~B})$. We obtained the periostin expression and matched it with the clinical information from six available data sets in GEO database. The results about the survival analysis supported our finding. The high-expression group had a better survival compared to the low-expression group ( $P=0.046, \mathrm{HR}=0.84,95 \% \mathrm{CI}=0.71-1$; Figure 4$)$.

To explore the association of periostin with different histologic subtypes of GC, the patients were divided into two groups according to the Lauren classification: intestinal type and diffuse type. In intestinal type GC, a significant difference in survival was observed between periostin epithelial cellpositive group and periostin epithelial cell-negative group $(P=0.006, \mathrm{HR}=0.505,95 \% \mathrm{CI}=0.306-0.831$; Figure $5 \mathrm{~A})$ and between high-expression subgroup and low-expression 
Table 2 Results of the clinicopathological parameters for gastric cancer with lymph node involvement by multivariate logistic analyses

\begin{tabular}{|c|c|c|c|c|}
\hline \multirow[b]{3}{*}{ Variables } & \multicolumn{4}{|c|}{ Lymph node involvement } \\
\hline & \multicolumn{2}{|l|}{ Total } & \multicolumn{2}{|c|}{ Epithelial cell-negative group } \\
\hline & OR $(95 \% \mathrm{Cl})$ & $P$-value & OR $(95 \% \mathrm{CI})$ & $P$-value \\
\hline $\begin{array}{l}\text { Periostin expression in epithelial cell or expression level in stroma } \\
\text { (positive vs negative or high level vs low level) }\end{array}$ & $0.494(0.268-0.909)$ & $<0.001$ & $0.935(0.522-1.676)$ & 0.028 \\
\hline Diameter $(\mathrm{cm} ; \geq 4$ vs $<4)$ & $2.375(1.393-4.050)$ & $<0.001$ & $2.925(1.611-5.310)$ & $<0.001$ \\
\hline Lauren type (diffuse type/intestinal type) & $1.578(0.907-2.744)$ & $<0.001$ & $\mathrm{I} .470(0.804-2.687)$ & $<0.001$ \\
\hline Serum CEA (ng/mL; $\geq 5$ vs $<5)$ & $3.549(1.534-8.211)$ & $<0.001$ & $3.416(1.274-9.156)$ & $<0.001$ \\
\hline Serum CAI $9-9$ (U/mL; $\geq 37$ vs $<37)$ & $1.737(0.819-3.685)$ & 0.003 & $1.387(0.606-3.172)$ & 0.031 \\
\hline Serum CA72-4 (U/mL; $\geq 6.9$ vs $<6.9)$ & $1.199(0.622-2.313)$ & 0.005 & $\mathrm{I} .252(0.60 \mathrm{I}-2.608)$ & 0.031 \\
\hline Depth of invasion (T3+T4 vs T0+TI+T2) & $4.387(2.545-7.562)$ & $<0.001$ & $3.369(1.864-6.088)$ & $<0.001$ \\
\hline Differentiation status (poorly vs well+moderately) & $1.897(1.066-3.377)$ & $<0.001$ & $1.911(1.012-3.607)$ & $<0.001$ \\
\hline
\end{tabular}

Abbreviations: CA19-9, carbohydrate antigen 19-9; CA72-4, carbohydrate antigen 72-4; CEA, carcinoembryonic antigen.

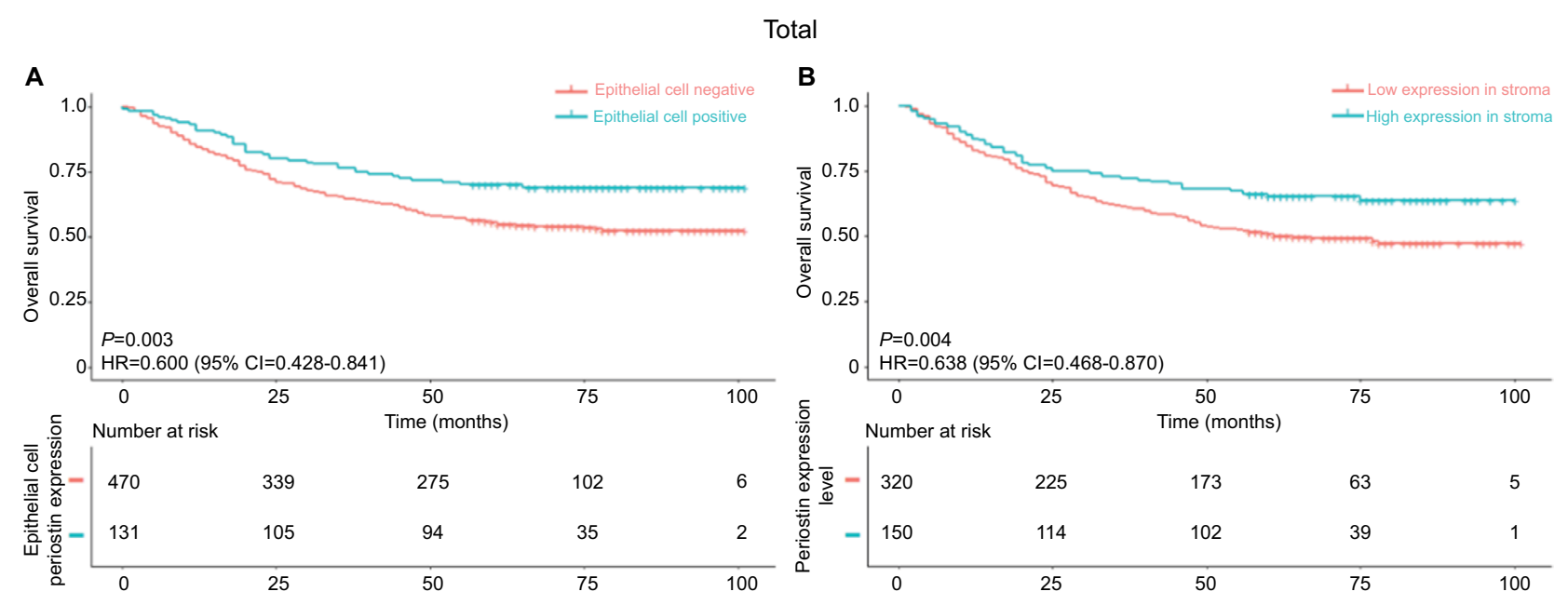

Figure 3 Periostin epithelial cell negative and low expression in epithelial cell-negative tissue were associated with the poor survival of GC patients.

Notes: (A) Different survivals between patients with periostin epithelial cell-negative or periostin epithelial cell-positive GC. (B) Different survivals between patients with stromal periostin low-expression or high-expression GC whose periostin was negative in epithelial cell tumors. Log-rank P-values are derived from Kaplan-Meier analysis with log-rank test.

Abbreviation: GC, gastric cancer.

subgroup in periostin epithelial cell-negative group $(P=0.018$, $\mathrm{HR}=0.561,95 \% \mathrm{CI}=0.344-0.913$; Figure 5B). There was no statistical significance with the diffuse type (Figure $5 \mathrm{C}$ and D). Multivariate Cox regression analysis showed that periostin expression in the epithelial cell positive tissues and high expression in epithelial cell-negative tissue was not an independent protection factor for intestinal type GC (Tables S2 and S3).

\section{Periostin recruited TAMs in diffuse type of GC}

TMAs were stained for CD163 to investigate the relevance between periostin expression and CD163(+) TAMs. In accordance with the previous study, in diffuse type GC, both epithelial cell-positive group and stromal periostin highexpression group had a higher relative intensity of CD163 than the other groups $(P=0.008$ and 0.008 , respectively; Figure 6C, D). However, periostin expression was not associated with CD163 in intestinal type GC (Figure 6A and B).

\section{Discussion}

In situ hybridization analysis showed that periostin mRNA was absent in lung cancer tissues but was expressed in the stromal tissue that surrounded the cancer tissue. ${ }^{16}$ There are two conflicting studies that have been published on the periostin production in the breast cancer; first study showed that periostin was produced by the epithelial cancer cells and the second study showed that periostin was produced 


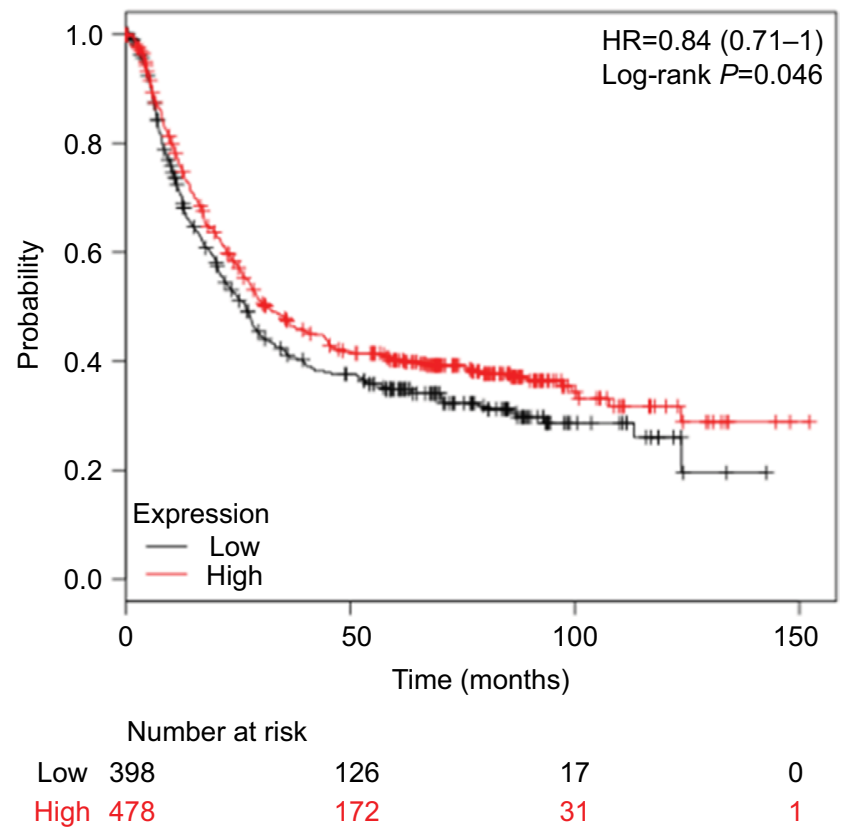

Figure 4 Different survivals between patients with different expression levels of periostin in GEO data sets, GSEI 42 I0, GSEI5459, GSE22377, GSE29272, GSE5 I I05, and GSE62254.

Note: Log-rank $P$-values are derived from Kaplan-Meier analysis with log-rank test. Abbreviation: GEO, Gene Expression Omnibus.

by the stromal cells around the cancer cells. ${ }^{34} \mathrm{Kim}$ et al ${ }^{13}$ stated that there are two explanations for these controversial findings; first, the function of periostin is dependent on the tumor histopathology. They found that periostin mRNA was overexpressed in urinary bladder squamous cell carcinoma, which was contrary in the urinary bladder transitional cell carcinoma. ${ }^{34}$ In our study, we found that in GC tissue, periostin expression in stroma was downregulated and periostin expression in epithelial cells was upregulated.

According to the Lauren Classification, GC can be histologically divided into two subtypes: the intestinal type and the diffused type.$^{35}$ Having differences in etiology, pathology, and epidemiology, the two subtypes of GC have certain genes or proteins that are expressed at different levels. ${ }^{35}$ We studied the difference in periostin expression between the two histological subtypes. We found that the stromal and (epithelial) cellular periostin expression levels were significantly higher in the intestinal type GC than the diffuse type GC $(P<0.001)$. This finding proved that periostin may play a different role in different histological types. Second, the function is also dependent on the spicing site at the $C$-terminal region. ${ }^{34,36}$ Takeshita et $a l^{36}$ concluded that five different spliced transcripts can be produced at the $C$-terminal region. Although their functions have not been studied, they speculated that the alternative splicing event might affect the invasiveness and metastasis function of tumors. Kim et $\mathrm{al}^{34}$ showed that $C$-terminal region of the periostin can suppress the in vitro cell metastasis and in vivo lung metastasis. Periostin stabilizes p53 and E-cadherin proteins through the Rb/E2F1/p14ARF/ $\mathrm{Mdm} 2$ signaling pathway, which suppresses the malignancy and the metastasis of tumor cells both in vivo and vitro. ${ }^{25} \mathrm{We}$ compared the survival of the patients with different periostin expression levels in GC. Results showed that patients in epithelial cell-positive group and high-expression stroma subgroup had a better survival. Analysis of 876 patients from six GEO data sets proved our result. We found that the expression of periostin was associated with a better survival.

The NCCN guidelines recommend special treatment strategy for patients with $\mathrm{LN}$ metastasis. The treatment should be either perioperative chemotherapy or chemoradiotherapy, but the diagnosis of LN metastasis should be performed accurately. ${ }^{23}$ During gastrectomy, the extended $\mathrm{LN}$ resection depends on the number of $\mathrm{LN}$ metastasis. Standard D2 lymphadenectomy is not recommended in all GC patients. ${ }^{37,38}$ In an Italian research, it has been suggested that D2 lymphadenectomy should be carried out only in advanced GC patients. ${ }^{39}$ Early GC can be managed by endoscopic treatment. ${ }^{40}$ Therefore, the prediction of LN metastasis is essential before the selection of the therapeutic options..$^{22}$ Nowadays, in clinical settings, LNs metastasis is mostly evaluated by imaging techniques such as endoscopic ultrasonography (EUS), computed tomography, and magnetic resonance imaging (MRI) ${ }^{41-43}$ Studies found that these imaging methods are not reliable to determine LNs metastasis due to their low sensitivity and specificity. ${ }^{41}$ This encourages researchers to search for novel biomarkers that can predict LNs metastasis in GC patients. ${ }^{44}$ From our study, we found that periostin was an independent predictor of LN metastasis. Patients with high periostin expression have less LN metastasis. Periostin may help clinician to preoperatively plan for extended LN dissection in GC patients.

Several evidences showed that the tumor microenvironment plays an important role in the tumor development. ${ }^{26}$ One of the major constituents of the tumor microenvironment is TAMs, which is also known as tumor-infiltrating macrophages. ${ }^{45-47}$ Studies showed that TAMs promote tumor initiation, development, and distant metastasis by enhancing the tumor angiogenesis, invasion, and migration ability. ${ }^{28}$ Macrophage is one of the major components that infiltrates most tumors. Macrophages exists in two polarized conditions, first is classically activated (M1) state and the second is alternatively activated (M2). ${ }^{48,49} \mathrm{M} 1$ macrophage has the antitumor 
Intestinal type

A
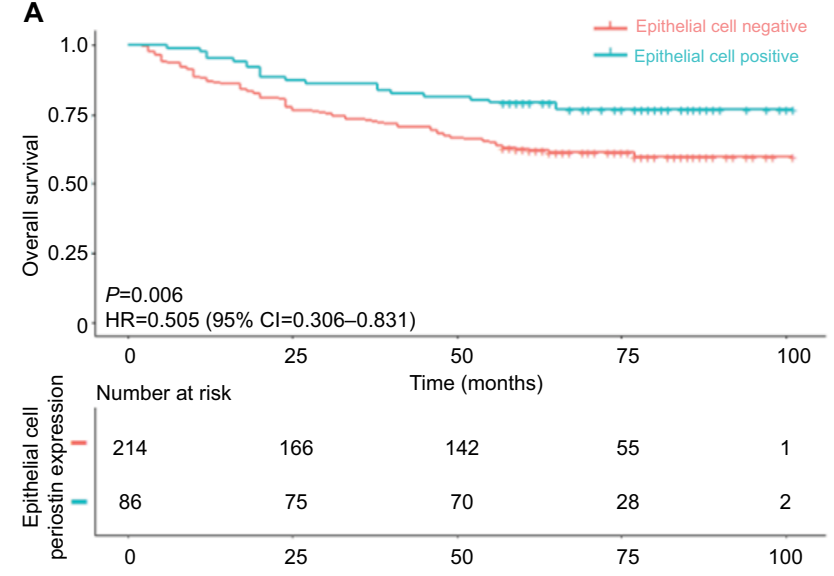

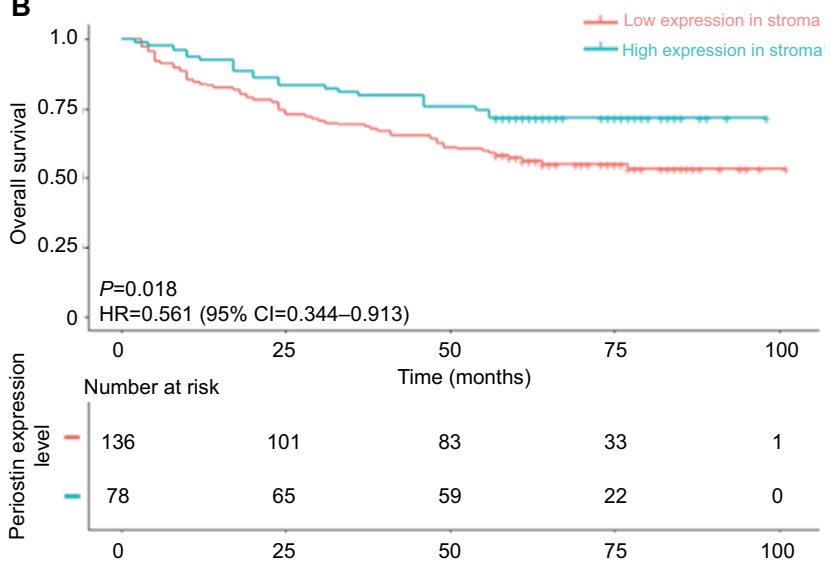

Diffuse type

C

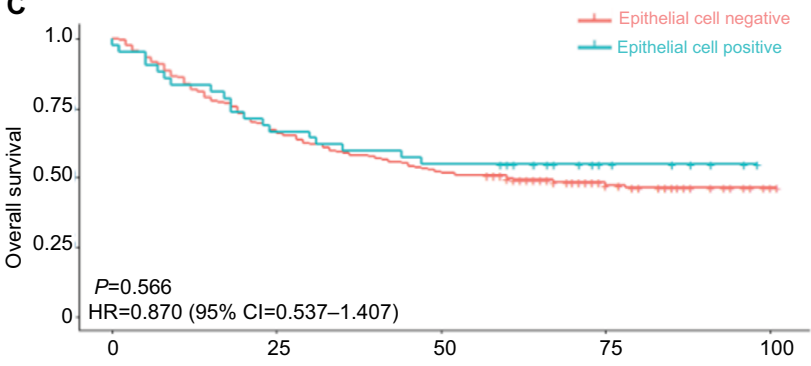

D

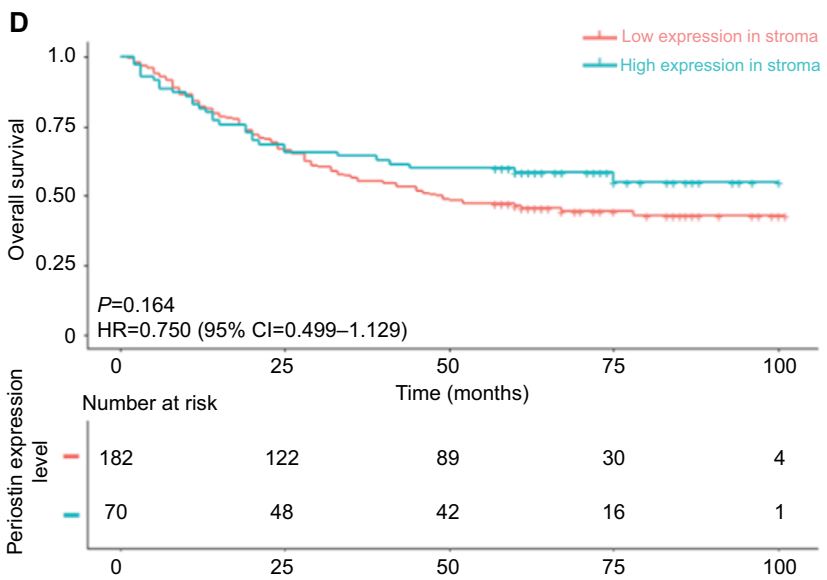

Figure 5 The loss of periostin expression is associated with worse survival of intestinal type GC patients compared to that of diffuse type GC patients.

Notes: (A and B) Different survivals between patients with intestinal type GC according to the cellular or stromal periostin expression. (C and D) Different survivals between patients with diffuse type GC according to the cellular or stromal periostin expression. Log-rank P-values are derived from Kaplan-Meier analysis with log-rank test. Abbreviation: GC, gastric cancer.

Intestinal type

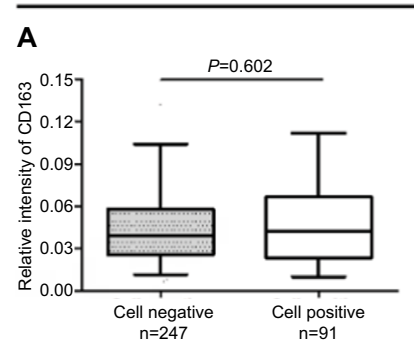

\section{B}

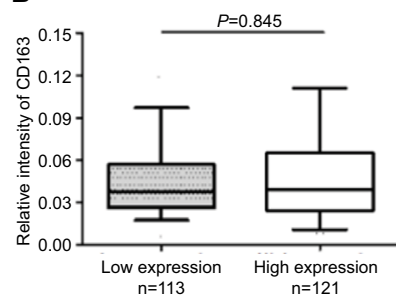

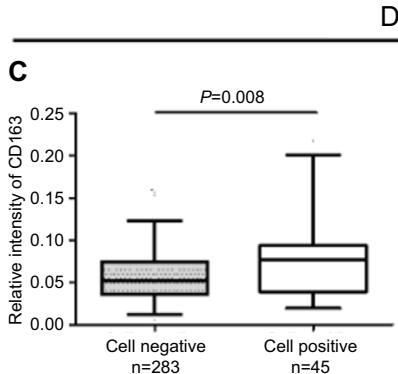

Diffuse type

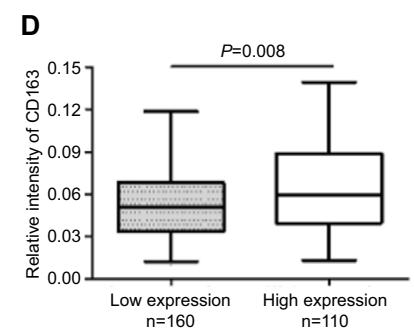

Figure 6 Expression of CDI63 in different periostin expression tumor tissues.

Notes: (A and B) Neither epithelial cellular periostin nor stromal periostin expression was associated with expression of CDI63 in intestinal type GC. (C and D) Epithelial cellular periostin and stromal periostin had a positive area/total correlation with CDI63 in diffuse type GC. Relative intensity of CDI63 was represented by positive area/ total area of the spot).

effect, whereas the M2 macrophage enhances tumor invasion and metastasis; most of the TAM have M2-like phenotype. ${ }^{48,49}$ There are several markers for M1 and M2 macrophages, and CD68 is a pan-macrophage marker that is frequently used. ${ }^{49,50}$ CD68 is a marker for TAM that recognizes both M1 and M2 macrophages. CD163 is a marker specifically for M2 macro- phages, and it has been used in several researchers to study aggressive cancers, and it is proved that CD163 is associated with poor overall survival in cancer patients. ${ }^{45}$ In breast cancer, tumor stroma infiltrated with CD163 macrophages was associated with high grade and tumor size. ${ }^{50}$ Our study showed that CD163 is associated with diffuse type GC. 
Intestinal GC is usually associated with lymphatic or vascular invasion, and intestinal type GC has a better survival than diffuse type GC. ${ }^{3}$ Diffuse GC usually originates from the gastric mucosa and mostly occurs in younger patients because of a chronic active inflammation..$^{35}$ Periostin has immunological roles in various malignancies and diseases. ${ }^{28,30,31}$ Studies found that periostin recruit macrophages through integrin alpha-v-beta-3. ${ }^{26}$ We studied the association of periostin and the tumor microenvironment. In diffuse type GC, the expression of periostin was associated with CD163(+) TAMs. This meant that in diffuse type GC patients, periostin might recruit CD163(+) TAMs. This can explain why periostin can act as a suppressor in intestinal type GC and not in diffuse type GC.

\section{Conclusion}

Extended lymphadenectomy is a very important decision for surgeons preparing for surgery. Periostin expression can help surgeons in predicting the LN metastasis and survival of patients. We found that high periostin expression has a more favorable survival in patients with intestinal GC. We also found that in diffuse GC, periostin might recruit CD163 (+) TAM, which might account for its poorer survival.

\section{Ethics statement}

Ethical approval was obtained from the ethics committee of the Second Affiliated Hospital of Wenzhou Medical University, and written informed consent was obtained from the patients whose tissue and medical data were used in this study.

\section{Acknowledgements}

This study was supported by Natural Science Foundation of China (81871966, 81572451, 81472308, 31470891), Zhejiang Provincial Natural Science Foundation of China (LY15H160062), and Wenzhou Municipal Science and Technology Bureau (Y20150054, Y20150057, Y20150125).

\section{Author contributions}

Teming Zhang generated all the data with the help of Arvine Chandoo and Xincheng Huang. Teming Zhang conducted the TMA IHC staining, and Xiangwei Sun analyzed all the IHC data with the help of all the authors. Arvine Chandoo performed all the statistical analyses and wrote the manuscript. Teming Zhang directed all the studies. All authors contributed to data analysis, drafting or revising the article, gave final approval of the version to be published, and agree to be accountable for all aspects of the work.

\section{Disclosure}

The authors report no conflicts of interest in this work.

\section{References}

1. Torre LA, Bray F, Siegel RL, Ferlay J, Lortet-Tieulent J, Jemal A. Global cancer statistics, 2012. CA Cancer J Clin. 2015;65(2):87-108.

2. Sun $\mathrm{C}, \mathrm{Zhao} \mathrm{X}, \mathrm{Xu} \mathrm{K}$, et al. Periostin: a promising target of therapeutical intervention for prostate cancer. J Transl Med. 2011;9:99.

3. Vauhkonen M, Vauhkonen H, Sajantila A, Sipponen P. Differences in genomic instability between intestinal- and diffuse-type gastric cancer. Gastric Cancer. 2005;8(4):238-244.

4. Qiu MZ, Cai MY, Zhang DS, et al. Clinicopathological characteristics and prognostic analysis of Lauren classification in gastric adenocarcinoma in China. J Transl Med. 2013;11:58.

5. Uchida M, Shiraishi H, Ohta S, et al. Periostin, a matricellular protein, plays a role in the induction of chemokines in pulmonary fibrosis. $\mathrm{Am}$ J Respir Cell Mol Biol. 2012;46(5):677-686.

6. Rios H, Koushik SV, Wang H, et al. Periostin null mice exhibit dwarfism, incisor enamel defects, and an early-onset periodontal disease-like phenotype. Mol Cell Biol. 2005;25(24):11131-11144.

7. Snider P, Hinton RB, Moreno-Rodriguez RA, et al. Periostin is required for maturation and extracellular matrix stabilization of noncardiomyocyte lineages of the heart. Circ Res. 2008;102(7):752-760.

8. Oka T, Xu J, Kaiser RA, et al. Genetic manipulation of periostin expression reveals a role in cardiac hypertrophy and ventricular remodeling. Circ Res. 2007;101(3):313-321.

9. Ruan K, Bao S, Ouyang G. The multifaceted role of periostin in tumorigenesis. Cell Mol Life Sci. 2009;66(14):2219-2230.

10. Skonier J, Neubauer M, Madisen L, Bennett K, Plowman GD, Purchio AF. cDNA cloning and sequence analysis of beta ig-h3, a novel gene induced in a human adenocarcinoma cell line after treatment with transforming growth factor-beta. DNA Cell Biol. 1992;11(7): 511-522.

11. Lebaron RG, Bezverkov KI, Zimber MP, Pavelec R, Skonier J, Purchio AF. [beta] IG-H3, a Novel Secretory Protein Inducible by Transforming Growth Factor-[beta], Is Present in Normal Skin and Promotes the Adhesion and Spreading of Dermal Fibroblasts In Vitro. J Invest Dermatol. 1995;104(5):844-849.

12. Skonier J, Bennett K, Rothwell V, et al. beta ig-h3: a transforming growth factor-beta-responsive gene encoding a secreted protein that inhibits cell attachment in vitro and suppresses the growth of $\mathrm{CHO}$ cells in nude mice. DNA Cell Biol. 1994;13(6):571-584.

13. Haertel-Wiesmann M, Liang Y, Fantl WJ, Williams LT. Regulation of cyclooxygenase- 2 and periostin by Wnt-3 in mouse mammary epithelial cells. J Biol Chem. 2000;275(41):32046-32051.

14. Sasaki H, Lo KM, Chen LB, et al. Expression of Periostin, homologous with an insect cell adhesion molecule, as a prognostic marker in nonsmall cell lung cancers. Jpn J Cancer Res. 2001;92(8):869-873.

15. Murakami D, Takamori S, Kawahara A, et al. Periostin Expression in Non-Small Cell Lung Cancer: Clinical Significance. Kurume Med J. 2018;64(1.2):13-20.

16. Sasaki H, Dai M, Auclair D, et al. Serum level of the periostin, a homologue of an insect cell adhesion molecule, in thymoma patients. Cancer Lett. 2001;172(1):37-42.

17. Bao S, Ouyang G, Bai X, et al. Periostin potently promotes metastatic growth of colon cancer by augmenting cell survival via the Akt/PKB pathway. Cancer Cell. 2004;5(4):329-339.

18. Michaylira CZ, Wong GS, Miller CG, et al. Periostin, a cell adhesion molecule, facilitates invasion in the tumor microenvironment and annotates a novel tumor-invasive signature in esophageal cancer. Cancer Res. 2010;70(13):5281-5292.

19. Baril P, Gangeswaran R, Mahon PC, et al. Periostin promotes invasiveness and resistance of pancreatic cancer cells to hypoxia-induced cell death: role of the beta4 integrin and the PI3k pathway. Oncogene. 2007;26(14):2082-2094. 
20. Choi KU, Yun JS, Lee IH, et al. Lysophosphatidic acid-induced expression of periostin in stromal cells: Prognostic relevance of periostin expression in epithelial ovarian cancer. Int J Cancer. 2011;128(2): $332-342$.

21. Tischler V, Fritzsche FR, Wild PJ, et al. Periostin is up-regulated in high grade and high stage prostate cancer. BMC Cancer. 2010;10:273.

22. Pang W, Lou N, Jin C, et al. Combination of preoperative platelet/ lymphocyte and neutrophil/lymphocyte rates and tumor-related factors to predict lymph node metastasis in patients with gastric cancer. Eur J Gastroenterol Hepatol. 2016;28(5):493-502.

23. Ajani JA, D'Amico TA, Almhanna K, et al. Gastric Cancer, Version 3.2016. J Natl Compr Canc Netw. 2016;14(10):1286-1312.

24. Kikuchi Y, Kunita A, Iwata C, et al. The niche component periostin is produced by cancer-associated fibroblasts, supporting growth of gastric cancer through ERK activation. Am J Pathol. 2014;184(3):859-870.

25. Lv H, Liu R, Fu J, et al. Epithelial cell-derived periostin functions as a tumor suppressor in gastric cancer through stabilizing p53 and E-cadherin proteins via the $\mathrm{Rb} / \mathrm{E} 2 \mathrm{~F} 1 / \mathrm{p} 14 \mathrm{ARF} / \mathrm{Mdm} 2$ signaling pathway. Cell Cycle. 2014;13(18):2962-2974.

26. Fessler E, Dijkgraaf FE, De Sousa E Melo F, Medema JP. Cancer stem cell dynamics in tumor progression and metastasis: is the microenvironment to blame? Cancer Lett. 2013;341(1):97-104.

27. Zhou W, Ke SQ, Huang Z, et al. Periostin secreted by glioblastoma stem cells recruits M2 tumour-associated macrophages and promotes malignant growth. Nat Cell Biol. 2015;17(2):170-182.

28. Tang M, Liu B, Bu X, Zhao P. Cross-talk between ovarian cancer cells and macrophages through periostin promotes macrophage recruitment. Cancer Sci. 2018;109(5):1309-1318.

29. Guo X, Xue H, Shao Q, et al. Hypoxia promotes glioma-associated macrophage infiltration via periostin and subsequent M2 polarization by upregulating TGF-beta and M-CSFR. Oncotarget. 2016;7(49):80521-80542.

30. Furudate S, Fujimura T, Kakizaki A, et al. The possible interaction between periostin expressed by cancer stroma and tumor-associated macrophages in developing mycosis fungoides. Exp Dermatol. 2016;25(2):107-112.

31. Fujimura T, Kakizaki A, Sato Y, Tanita K, Furudate S, Aiba S. The Immunological Roles of Periostin/Tumor-Associated Macrophage Axis in Development of Dermatofibrosarcoma Protuberans. Anticancer Res. 2017;37(6):2867-2873.

32. Fujimura T, Kakizaki A, Furudate S, Aiba S. A possible interaction between periostin and $\mathrm{CD}_{163^{+}}$skin-resident macrophages in pemphigus vulgaris and bullous pemphigoid. Exp Dermatol. 2017;26(12):1193-1198.

33. Xu JF, Zhao ZG, Ye LL, et al. Prognostic significance of Daxx NCR (Nuclear/Cytoplasmic Ratio) in gastric cancer. Cancer Med. 2017;6(9):2063-2075.

34. Kim CJ, Yoshioka N, Tambe Y, Kushima R, Okada Y, Inoue H. Periostin is down-regulated in high grade human bladder cancers and suppresses in vitro cell invasiveness and in vivo metastasis of cancer cells. Int $J$ Cancer. 2005;117(1):51-58.
35. Ma J, Shen H, Kapesa L, Zeng S. Lauren classification and individualized chemotherapy in gastric cancer. Oncol Lett. 2016;11(5):2959-2964.

36. Takeshita S, Kikuno R, Tezuka K, Amann E. Osteoblast-specific factor 2: cloning of a putative bone adhesion protein with homology with the insect protein fasciclin I. Biochem J. 1993;294(Pt 1): 271-278.

37. Cuschieri A, Weeden S, Fielding J, et al. Patient survival after D1 and D2 resections for gastric cancer: long-term results of the MRC randomized surgical trial. Surgical Co-operative Group. Br J Cancer. 1999;79(9-10):1522-1530.

38. Schuhmacher C, Gretschel S, Lordick F, et al. Neoadjuvant chemotherapy compared with surgery alone for locally advanced cancer of the stomach and cardia: European Organisation for Research and Treatment of Cancer randomized trial 40954. J Clin Oncol. 2010;28(35):5210-5218.

39. Degiuli M, Sasako M, Ponti A, et al; Italian Gastric Cancer Study Group. Randomized clinical trial comparing survival after D1 or D2 gastrectomy for gastric cancer. Br J Surg. 2014;101(2):23-31.

40. Feng XY, Wang W, Luo GY, et al. Comparison of endoscopic ultrasonography and multislice spiral computed tomography for the preoperative staging of gastric cancer - results of a single institution study of 610 Chinese patients. PLoS One. 2013;8(11):e78846.

41. Kwee RM, Kwee TC. Imaging in assessing lymph node status in gastric cancer. Gastric Cancer. 2009;12(1):6-22.

42. Seevaratnam R, Cardoso R, McGregor C, et al. How useful is preoperative imaging for tumor, node, metastasis (TNM) staging of gastric cancer? A meta-analysis. Gastric Cancer. 2012;15 Suppl 1:S3-S18.

43. Shim CN, Lee SK. Endoscopic submucosal dissection for undifferentiated-type early gastric cancer: do we have enough data to support this? World J Gastroenterol. 2014;20(14):3938-3949.

44. Jiang N, Deng JY, Liu Y, Ke B, Liu HG, Liang H. The role of preoperative neutrophil-lymphocyte and platelet-lymphocyte ratio in patients after radical resection for gastric cancer. Biomarkers. 2014;19(6):444-451.

45. Tang X, Mo C, Wang Y, Wei D, Xiao H. Anti-tumour strategies aiming to target tumour-associated macrophages. Immunology. 2013;138(2):93-104.

46. Hao NB, Lü MH, Fan YH, Cao YL, Zhang ZR, Yang SM. Macrophages in Tumor Microenvironments and the Progression of Tumors. Clin Dev Immunol. 2012;2012(11):1-11.

47. Shi C, Pamer EG. Monocyte recruitment during infection and inflammation. Nat Rev Immunol. 2011;11(11):762-774.

48. Maniecki MB, Etzerodt A, Ulhøi BP, et al. Tumor-promoting macrophages induce the expression of the macrophage-specific receptor CD163 in malignant cells. Int J Cancer. 2012;131(10):2320-2331.

49. Richards DM, Hettinger J, Feuerer M. Monocytes and macrophages in cancer: development and functions. Cancer Microenviron. 2013;6(2):179-191.

50. Medrek C, Pontén F, Jirström K, Leandersson K. The presence of tumor associated macrophages in tumor stroma as a prognostic marker for breast cancer patients. BMC Cancer. 2012;12:306. 


\section{Supplementary materials}

Table SI Univariate analysis of clinicopathological characteristics according to the lymph node involvement

\begin{tabular}{|c|c|c|c|c|c|c|}
\hline \multirow[t]{2}{*}{ Variables, $\mathbf{n}(\%)$} & \multicolumn{3}{|l|}{ Total } & \multicolumn{3}{|c|}{ Epithelial cell-negative group } \\
\hline & $\begin{array}{l}\text { Node-negative } \\
\text { group }(n=282)\end{array}$ & $\begin{array}{l}\text { Node-positive } \\
\text { group }(n=440)\end{array}$ & $P$-value & $\begin{array}{l}\text { Node-negative } \\
\text { group }(n=185)\end{array}$ & $\begin{array}{l}\text { Node-positive } \\
\text { group }(n=333)\end{array}$ & $P$-value \\
\hline $\begin{array}{l}\text { Periostin in epithelial cell or expression } \\
\text { level in stroma }\end{array}$ & & & $<0.001$ & & & 0.002 \\
\hline Negative or low level & $185(72.3)$ & $333(83.7)$ & & III (60.3) & $245(73.6)$ & \\
\hline Positive or high level & $71(27.7)$ & $65(16.3)$ & & $73(39.7)$ & $88(26.4)$ & \\
\hline Gender & & & 0.776 & & & 0.834 \\
\hline Male & $204(72.3)$ & $314(7 \mid .4)$ & & $126(68.5)$ & $231(69.4)$ & \\
\hline Female & $78(27.7)$ & $126(28.6)$ & & $58(3 \mid .5)$ & $102(30.6)$ & \\
\hline Age & & & $0.88 \mid$ & & & 0.928 \\
\hline$<60$ & $145(52.0)$ & $227(52.5)$ & & $99(54.1)$ & $175(53.7)$ & \\
\hline$\geq 60$ & $134(48.0)$ & $205(47.5)$ & & $84(45.9)$ & $15 \mid(46.3)$ & \\
\hline Diameter $(\mathrm{cm})$ & & & $<0.001$ & & & $<0.001$ \\
\hline$<4$ & $199(71.3)$ & $135(30.8)$ & & $131(72.0)$ & $101(30.4)$ & \\
\hline$\geq 4$ & $80(28.7)$ & $304(69.2)$ & & $5 \mathrm{I}(28.0)$ & $231(69.6)$ & \\
\hline Lauren type & & & $<0.001$ & & & $<0.001$ \\
\hline Intestinal type & $162(63.0)$ & $170(4 \mid .2)$ & & $107(59.4)$ & $132(39.8)$ & \\
\hline Diffuse type & $95(37.0)$ & $243(58.8)$ & & $73(40.6)$ & $200(60.2)$ & \\
\hline Serum CEA (ng/mL) & & & $<0.001$ & & & $<0.001$ \\
\hline$<5$ & 247 (9I.5) & $305(74.0)$ & & $166(93.8)$ & $240(76.4)$ & \\
\hline$\geq 5$ & $23(8.5)$ & $107(26.0)$ & & II (6.2) & $74(23.6)$ & \\
\hline Serum CAI9-9 (U/mL) & & & $<0.001$ & & & $<0.001$ \\
\hline$<37$ & $240(93.0)$ & $310(78.5)$ & & $156(92.3)$ & $237(79.0)$ & \\
\hline$\geq 37$ & $18(7.0)$ & $85(21.5)$ & & $13(7.7)$ & $63(21.0)$ & \\
\hline Serum CA72-4 (U/mL) & & & $<0.001$ & & & 0.002 \\
\hline$<6.9$ & $17 \mid(88.1)$ & $234(72.9)$ & & III (87.4) & $183(73.5)$ & \\
\hline$\geq 6.9$ & $23(11.9)$ & $87(27.1)$ & & $16(12.6)$ & $66(26.5)$ & \\
\hline Depth of invasion & & & $<0.001$ & & & $<0.001$ \\
\hline $\mathrm{T} 0+\mathrm{TI}+\mathrm{T} 2$ & $193(68.7)$ & $75(17.1)$ & & $120(65.2)$ & $56(16.8)$ & \\
\hline $\mathrm{T} 3+\mathrm{T} 4$ & $88(3 \mid .3)$ & $364(82.9)$ & & $64(34.8)$ & $277(83.2)$ & \\
\hline Differentiation status & & & $<0.001$ & & & $<0.001$ \\
\hline Well and moderate & $112(46.3)$ & $112(26.7)$ & & $68(43.0)$ & $78(24.9)$ & \\
\hline Poor and undifferentiated & $130(53.7)$ & $307(73.3)$ & & $90(57.0)$ & $235(75.1)$ & \\
\hline
\end{tabular}

Abbreviations: CA19-9, carbohydrate antigen 19-9; CA72-4, carbohydrate antigen 72-4; CEA, carcinoembryonic antigen.

Table S2 Cox regression analysis of periostin expression in epithelial cell according to the overall survival in patients with intestinal type gastric cancer

\begin{tabular}{|c|c|c|c|c|}
\hline \multirow[t]{3}{*}{ Variables } & \multicolumn{4}{|l|}{ Overall survival } \\
\hline & \multicolumn{2}{|l|}{ Univariate analysis } & \multicolumn{2}{|l|}{ Multivariate analysis } \\
\hline & HR (95\% CI) & $P$-value & HR (95\% Cl) & $P$-value \\
\hline Periostin expression in epithelial cell (positive vs negative) & $0.505(0.306-0.831)$ & 0.007 & $0.884(0.454-1.724)$ & 0.718 \\
\hline Adjuvant chemotherapy (yes vs no) & $3.472(2.109-5.716)$ & $<0.001$ & $1.405(0.7 \mid 4-2.762)$ & 0.325 \\
\hline Serum CEA $(\mathrm{ng} / \mathrm{mL} ; \geq 5$ vs $<5)$ & $2.646(1.733-4.042)$ & $<0.001$ & $\mathrm{I} .077(0.58 \mathrm{I}-\mathrm{I} .997)$ & 0.814 \\
\hline Serum CA I9-9 (U/mL; $\geq 37$ vs <37) & $2.555(1.633-3.999)$ & $<0.001$ & $1.535(0.825-2.765)$ & 0.154 \\
\hline Serum CA72-4 (U/mL; $\geq 6.9$ vs $<6.9)$ & $1.945(1.157-3.268)$ & 0.012 & $1.098(0.617-1.956)$ & 0.750 \\
\hline Diameter $(\mathrm{cm} ; \geq 4$ vs $<4)$ & $4.408(2.838-6.848)$ & $<0.001$ & $2.228(1.213-4.091)$ & 0.010 \\
\hline Depth of invasion (T3+T4 vs $\mathrm{T} 0+\mathrm{TI}+\mathrm{T} 2)$ & $7.999(4.546-\mid 4.075)$ & $<0.001$ & $3.220(\mathrm{I} .278-8.1 \mathrm{II})$ & 0.013 \\
\hline Lymph node involvement (yes vs no) & $7.094(4.156-12.109)$ & $<0.001$ & $3.917(|.499-| 0.231)$ & 0.005 \\
\hline TNM stage $(I I I+I V$ vs $0+I+I I)$ & $6.302(4.07 I-9.756)$ & $<0.001$ & $0.589(0.240-1.445)$ & 0.247 \\
\hline Differentiation grade (poorly vs well+moderately) & $1.434(0.967-2.127)$ & 0.073 & I.29I (0.77I-2.I59) & 0.331 \\
\hline
\end{tabular}

Abbreviations: CA19-9, carbohydrate antigen 19-9; CA72-4, carbohydrate antigen 72-4; CEA, carcinoembryonic antigen. 
Table S3 Cox regression analysis of periostin expression in stroma according to the overall survival in the epithelial cell-negative patients with intestinal gastric cancer

\begin{tabular}{|c|c|c|c|c|}
\hline \multirow[t]{3}{*}{ Variables } & \multicolumn{4}{|l|}{ Overall survival } \\
\hline & \multicolumn{2}{|l|}{ Univariate analysis } & \multicolumn{2}{|c|}{ Multivariate analysis } \\
\hline & HR (95\% CI) & $P$ value & HR (95\% CI) & $P$ value \\
\hline Periostin expression in stroma (high level vs low level) & $0.561(0.344-0.913)$ & 0.020 & $0.696(0.38 I-I .270)$ & 0.237 \\
\hline Adjuvant chemotherapy (yes vs no) & $3.124(1.809-5.395)$ & $<0.001$ & $1.543(0.729-3.265)$ & 0.257 \\
\hline Serum CEA (ng/mL; $\geq 5$ vs $<5)$ & $3.071(1.917-4.920)$ & $<0.001$ & $1.369(0.704-2.665)$ & 0.355 \\
\hline Serum CAI9-9 (U/mL; $\geq 37$ vs <37) & $2.349(1.429-3.859)$ & 0.001 & $1.497(0.777-2.886)$ & 0.228 \\
\hline Serum CA72-4 (U/mL; $\geq 6.9$ vs <6.9) & $1.638(0.911-2.944)$ & 0.099 & $0.997(0.520-1.911)$ & 0.994 \\
\hline Diameter $(\mathrm{cm} ; \geq 4$ vs $<4)$ & $3.999(2.469-6.479)$ & $<0.001$ & $1.949(1.014-3.746)$ & 0.045 \\
\hline Depth of invasion (T3+T4 vs T0+TI+T2) & $6.032(3.331-10.922)$ & $<0.001$ & $2.162(0.838-5.581)$ & 0.111 \\
\hline Lymph node involvement (yes vs no) & $4.856(2.77 \mathrm{I}-8.508)$ & $<0.001$ & $2.716(0.980-7.523)$ & 0.055 \\
\hline TNM stage $(\mathrm{III}+\mathrm{IV}$ vs $0+\mathrm{I}+\mathrm{II})$ & $4.633(2.890-7.427)$ & $<0.001$ & $0.710(0.270-1.867)$ & 0.487 \\
\hline Differentiation grade (poorly vs well+moderately) & $1.249(0.803-1.94 I)$ & 0.324 & I.347 (0.765-2.372) & 0.302 \\
\hline
\end{tabular}

Abbreviations: CA19-9, carbohydrate antigen 19-9; CA72-4, carbohydrate antigen 72-4; CEA, carcinoembryonic antigen.

\section{Publish your work in this journal}

Cancer Management and Research is an international, peer-reviewed open access journal focusing on cancer research and the optimal use of preventative and integrated treatment interventions to achieve improved outcomes, enhanced survival and quality of life for the cancer patient. The manuscript management system is completely online and includes a very quick and fair peer-review system, which is all easy to use. Visit http://www.dovepress.com/testimonials.php to read real quotes from published authors. 